\title{
A DEFORMATION LEMMA AND SOME CRITICAL POINT THEOREMS
}

\author{
YIHONG DU
}

\begin{abstract}
A new deformation lemma for functions satisfying the Palais-Smale condition on a real Banach space is obtained. This is used to deduce some critical point theorems which are extensions of some well known results.
\end{abstract}

\section{A DeFormation LemMa}

Throughout this paper, we assume that $X$ is a real Banach space, $f \in C^{1}\left(X, R^{1}\right)$ satisfies the Palais-Smale condition: any sequence $\left(x_{n}\right)$ for which $f\left(x_{n}\right)$ is bounded and $f^{\prime}\left(x_{n}\right) \rightarrow 0$ possesses a converging subsequence. For a real number $c$, define

and

$$
\begin{gathered}
f_{c}=\{x \in X: f(x) \leqslant c\}, f^{c}=\{x \in X: f(x) \geqslant c\} \\
K_{c}=\left\{x \in X: f(x)=c \text { and } f^{\prime}(x)=0\right\} .
\end{gathered}
$$

Lemma 1.1. Let $A, B$ be two closed sets in $X$ with $A \subset f^{c}, B \subset f_{c}$ and $A \cap B=\emptyset$. Then, if $B \cap K_{c}=\emptyset$, we can find an $\varepsilon>0$ and a homeomorphism $\eta$ of $X$ onto $X$ such that

(i) $f(\eta x) \leqslant f(x)$ for all $x \in X$;

(ii) $\eta x=x$ and hence $f(\eta x)=f(x)$ for $x \in A$;

(iii) $f(\eta x) \leqslant c-\varepsilon$ for $x \in B$.

Proof: Since $f$ satisfies the Palais-Smale condition, $K_{c}$ is a compact set or an empty set. Hence from $B \cap K_{c}=\emptyset$ we can find a $\delta_{1}>0$ such that $\bar{U}_{3 \delta_{1}}(B) \cap K_{c}=\emptyset$, where $U_{\tau}(B)$ denotes the $\tau$-neighbourhood of $B$ and $\bar{U}_{3 \delta_{1}}(B)$ is the closure of $U_{3 \delta_{1}}(B)$. Using the Palais-Smale condition again we find $\varepsilon_{1}>0, \sigma_{1}>0$ such that

$$
\left\|f^{\prime}(x)\right\| \geqslant \sigma_{1} \text { for } x \in \bar{U}_{3 \sigma_{1}}(B) \cap f^{c-3 \varepsilon_{1}} \cap f_{c+3 e_{1}}
$$

Received 20 March 1990

After seeing the original manuscript, Professor P.H. Rabinowitz sent me some suggestions which motivated my further work on this line; he also sent me his work [10] by which I could clear up several mistakes in the original manuscript. I wish to express my gratitude here to Professor P.H. Rabinowitz for his kind help. I also wish to thank Mr. Guijie Qi for some helpful conversations.

Copyright Clearance Centre, Inc. Serial-fee code: 0004-9729/91 \$A2.00+0.00. 
Now for $x \in X$, define

$$
\phi(x)=\frac{d\left(x, X \backslash\left(U_{2 \delta_{1}} \cap f^{c-2 \varepsilon_{1}} \cap f_{c+2 e_{1}}\right)\right)}{d\left(x, X \backslash\left(U_{2 \delta_{1}} \cap f^{c-2 \varepsilon_{1}} \cap f_{c+2 e_{1}}\right)\right)+\left(d\left(x, U_{\delta_{1}} \cap f^{c-\varepsilon_{1}} \cap f_{c+\varepsilon_{1}}\right)\right)} .
$$

It is easy to check that $\phi: X \rightarrow[0,1]$ is locally Lipschitz continuous, $\phi(x)=0$ when $x \in X \backslash\left(U_{2 \delta_{1}}(B) \cap f^{c-2 \varepsilon_{1}} \cap f_{c+2 \varepsilon_{1}}\right)$, and $\phi(x)=1$ when $x \in U_{\delta_{1}}(B) \cap f^{c-\varepsilon_{1}} \cap f_{c+\varepsilon_{1}}$.

Let

$$
\begin{aligned}
h(s) & = \begin{cases}1, & \text { when } 0 \leqslant s \leqslant 1 \\
s^{-1}, & \text { when } s>1 .\end{cases} \\
w_{1}(x) & = \begin{cases}-\delta_{1} \phi(x) h(\|v(x)\|) v(x) & \text { for } x \in \bar{U}_{3 \delta_{1}}(P) \cap f^{c-3 \varepsilon_{1}} \cap f_{c+3 \varepsilon_{1}} \\
\theta & \text { for } x \in X \backslash\left(U_{3 \delta_{1}}(B) \cap f^{c-3 \varepsilon_{1}} \cap f_{c+3 \varepsilon_{1}}\right),\end{cases}
\end{aligned}
$$

where $v(x)$ is a pseudo-gradient of $f(x)$. It is easily seen that $w_{1}(x)$ is locally Lipschitz continuous, $\left\|w_{1}(x)\right\| \leqslant \delta_{1}$ for all $x \in X$, and that for $x \in \bar{U}_{\delta_{1}}(B) \cap f^{c-e_{1}} \cap f_{c+e_{1}}$, $w_{1}(x)=-\delta_{1} h(\|v(x)\|) v(x)$ and

$$
\begin{aligned}
-\left(f^{\prime}(x), w_{1}(x)\right) & =\delta_{1} h(\|v(x)\|)\left(f^{\prime}(z), v(x)\right) \\
& \geqslant \frac{1}{2} \delta_{1} h\left(2\left\|f^{\prime}(x)\right\|\right) 2\left\|f^{\prime}(x)\right\| \cdot\left\|f^{\prime}(x)\right\| \\
& \geqslant \frac{1}{2} \delta_{1} \min \left(1, \sigma_{1}\right) \sigma_{1} \triangleq \sigma>0 .
\end{aligned}
$$

Let $\xi(t)=\xi(t, x)$ be the unique solution of the problem

$$
\left\{\begin{aligned}
d \xi / d t & =w_{1}(\xi) \\
\xi(0) & =x
\end{aligned}\right.
$$

It is known from the theory of differential equations in Banach spaces that $\xi(t, x)$ is defined for all $(t, x) \in R^{1} \times X$. Let $B_{1}=\{\xi(t, x): x \in B, t \in[0,1]\}$. We show that (a) $B_{1}$ is a closed set and (b) $A \cap B_{1}=\emptyset$.

In fact, suppose $y_{n} \in B_{1}$ and $y_{n} \rightarrow y_{0}$. Then we can find $x_{n} \in B$ and $t_{n} \in[0,1]$ such that $y_{n}=\xi\left(t_{n}, x_{n}\right)$. Now $\left\{t_{n}\right\}$ must have a converging subsequence: $t_{n_{k}} \rightarrow t_{0} \in$ $[0,1]$, so let $z_{k}=\xi\left(t_{0}, x_{n_{k}}\right)$ and we have

$$
\begin{aligned}
\left\|z_{k}-y_{n_{k}}\right\| & =\left\|\xi\left(t_{0}, x_{n_{k}}\right)-\xi\left(t_{n_{k}}, x_{n_{k}}\right)\right\| \\
& =\left\|\int_{t_{n_{k}}}^{t_{0}} w_{1}\left(\xi\left(t, x_{n_{k}}\right)\right) d t\right\| \\
& \leqslant \delta_{1}\left|t_{0}-t_{n_{k}}\right| \rightarrow 0 .
\end{aligned}
$$


Hence $z_{k} \rightarrow y_{0}$ and $x_{n_{k}}=\xi\left(-t_{0}, z_{k}\right) \rightarrow \xi\left(-t_{0}, y_{0}\right)$ due to the continuous dependence of solutions on initial values. Let $x_{0}=\xi\left(-t_{0}, y_{0}\right)$; then since $x_{n_{k}} \rightarrow x_{0}, x_{n_{k}} \in B$ and $B$ is closed, we see that $x_{0} \in B$ and thus $y_{0}=\xi\left(t_{0}, x_{0}\right) \in B_{1}$. This proves the closedness of $B_{1}$.

Now we prove assertion (b). From the definition of $w_{1}(x)$, it is easy to check that $(d / d t) f(\xi(t, x)) \leqslant 0$ for $x \in X$ and $t \in R^{1}$. This implies that $f(\xi(t, x))$ is a decreasing function of $t$. Suppose by contradiction that $A \cap B_{1} \neq \emptyset$; then there are $x_{1} \in B$ and $t_{1} \in[0,1]$ such that $\xi\left(t_{1}, x_{1}\right) \in A$. Since $A \cap B=\emptyset$, we must have $t_{1}>0$. Using $A \subset f^{c}$ and $B \subset f_{c}$ we get for $t \in\left[0, t_{1}\right]$ that

$$
c \leqslant f\left(\xi\left(t_{1}, x_{1}\right)\right) \leqslant f\left(\xi\left(t, x_{1}\right)\right) \leqslant f\left(\xi\left(0, x_{1}\right)\right)=f\left(x_{1}\right) \leqslant c
$$

which implies

$$
f\left(\xi\left(t, x_{1}\right)\right)=c \text { for } t \in\left[0, t_{1}\right]
$$

On the other hand, we have for $t \in[0,1]$ that

$$
\left\|\xi\left(t, x_{1}\right)-x_{1}\right\|=\left\|\xi\left(t, x_{1}\right)-\xi\left(0, x_{1}\right)\right\|=\left\|\int_{0}^{t} w_{1}\left(\xi\left(s, x_{1}\right)\right) d s\right\| \leqslant \delta_{1} .
$$

Hence $\left\{\xi\left(t, x_{1}\right): t \in\left[0, t_{1}\right]\right\} \subset \bar{U}_{\delta_{1}}(B) \cap f^{c-\varepsilon_{1}} \cap f_{c+\varepsilon_{1}}$. Now using (1) we see that

$$
\begin{aligned}
\left(f^{\prime}\left(\xi\left(t, x_{1}\right)\right), w_{1}\left(\xi\left(t, x_{1}\right)\right)\right) & \leqslant-\sigma \text { for } t \in\left[0, t_{1}\right] \\
f\left(\xi\left(t_{1}, x_{1}\right)\right) & =f\left(\xi\left(0, x_{1}\right)\right)+\int_{0}^{t_{1}} \frac{d}{d t} f\left(\xi\left(t, x_{1}\right)\right) d t \\
& =f\left(x_{1}\right)+\int_{0}^{t_{1}}\left(f^{\prime}\left(\xi\left(t, x_{1}\right)\right), w_{1}\left(\xi\left(t, x_{1}\right)\right)\right) d t \\
& \leqslant f\left(x_{1}\right)-t_{1} \sigma<c
\end{aligned}
$$

which contradicts (2). Thus assertion (b) is proved.

For $x \in X$, define

$$
\psi_{1}(x)=\frac{d(x, A)}{d(x, A)+d\left(x, B_{1}\right)} .
$$

It is clear that $\psi_{1}: X \rightarrow[0,1]$ is continuous, so $A_{1}=\left\{x \in X: \psi_{1}(x) \leqslant \frac{1}{2}\right\}$ is a closed set. Evidently $A \subset \AA_{1}$ and $A_{1} \cap B_{1}=\emptyset$. Now for $x \in X$ define

$$
\psi(x)=\frac{d\left(x, A_{1}\right)}{d\left(x, A_{1}\right)+d\left(x, B_{1}\right)} .
$$


Clearly, $\psi: X \rightarrow[0,1]$ is locally Lipschitz continuous and $\psi(x)=\left\{\begin{array}{ll}0 & \text { for } x \in A_{1}, \\ 1 & \text { for } x \in B_{1}\end{array}\right.$. Let

$$
w(x)=\psi(x) w_{1}(x) \text { for all } x \in X,
$$

then $w(x)$ is locally Lipschitz continuous; $\|w(x)\| \leqslant \delta_{1}$ for all $x \in X ; w(x)=\theta$ for $x \in A_{1}$ and $w(x)=w_{1}(x)$ for $x \in B_{1}$.

Let $\eta(t)=\eta(t, x)$ be the unique solution of the problem

$$
\left\{\begin{array}{c}
d \eta / d t=w(\eta), \\
\eta(0)=x,
\end{array}\right.
$$

and then $\eta(t, x)$ is defined for all $(t, x) \in R^{1} \times X$. Define

$$
\varepsilon=\min \left(\sigma, \varepsilon_{1}\right) \text { and } \eta x=\eta(1, x) \text { for all } x \in X .
$$

We show that $\varepsilon, \eta$ meet our requirements (i) - (iii).

In fact, it is known from the theory of differential equations in Banach spaces that $\eta$ is a homeomorphism of $X$ onto $X$. We can easily check that $(d / d t) f(\eta(t, x)) \leqslant 0$, so $f(\eta(t, x))$ is a decreasing function of $t$. This gives $f(\eta(1, x)) \leqslant f(\eta(0, x))$, that is, $f(\eta x) \leqslant f(x)$ and (i) is satisfied. Since $w(x)=\theta$ for $x \in A_{1}$, we have $\eta(t, x)=x$ for all $t$ when $x \in \stackrel{\circ}{A}_{1}$; in particular, $\eta(1, x)=x$ for $x \in A$, that is, $\eta x=x$ for $x \in A$ and (ii) is proved.

Suppose by contradiction that (iii) is not satisfied. Then we find $x_{0} \in B$ such that $f\left(\eta x_{0}\right)>c-\varepsilon$. Let $y_{0}=\eta x_{0}$; then $y_{0}=\eta\left(1, x_{0}\right)$ and $f\left(y_{0}\right)>c-\varepsilon$. Since $\xi\left(t, x_{0}\right) \in B_{1}$ for $t \in[0,1]$ and $w(x)=w_{1}(x)$ when $x \in B_{1}$, we see that $w\left(\xi\left(t, x_{0}\right)\right)=w_{1}\left(\xi\left(t, x_{0}\right)\right)$ for $t \in[0,1]$. Hence $\xi(t)=\xi\left(t, x_{0}\right)$ satisfies $(3)$ for $t \in[0,1]$ and $x=x_{0}$. This implies $\xi\left(t, x_{0}\right)=\eta\left(t, x_{0}\right)$ for $t \in[0,1]$.

Now from $B \subset f_{c}$ we have for $t \in[0,1]$ that

$$
c \geqslant f\left(x_{0}\right)=f\left(\eta\left(0, x_{0}\right)\right) \geqslant f\left(\eta\left(t, x_{0}\right)\right) \geqslant f\left(\eta\left(1, x_{0}\right)\right)=f\left(y_{0}\right)>c-\varepsilon .
$$

So $\left\{\eta\left(t, x_{0}\right): t \in[0,1]\right\} \subset f^{c-\varepsilon} \cap f_{c}$. Moreover, for $t \in[0,1]$,

$$
\begin{aligned}
\left\|\eta\left(t, x_{0}\right)-x_{0}\right\| & =\left\|\eta\left(t, x_{0}\right)-\eta\left(0, x_{0}\right)\right\| \\
& =\left\|\int_{0}^{t}(d / d s) \eta\left(s, x_{0}\right) d s\right\|=\left\|\int_{0}^{t} w\left(\eta\left(s, x_{0}\right)\right) d s\right\| \leqslant \delta_{1} .
\end{aligned}
$$

Hence $\left\{\eta\left(t, x_{0}\right): t \in[0,1]\right\} \subset \bar{U}_{\delta_{1}}(B) \cap f^{c-e_{1}} \cap f_{c+e_{1}}$. Now using (1) and $w\left(\eta\left(t, x_{0}\right)\right)=$ 
$w_{1}\left(\eta\left(t, x_{0}\right)\right)$ for $t \in[0,1]$ we get

$$
\begin{aligned}
f\left(x_{0}\right)-f\left(y_{0}\right) & =f\left(\eta\left(0, x_{0}\right)\right)-f\left(\eta\left(1, x_{0}\right)\right) \\
& =-\int_{0}^{1} \frac{d}{d t} f\left(\eta\left(t, x_{0}\right)\right) d t \\
& =-\int_{0}^{1}\left(f^{\prime}\left(\eta\left(t, x_{0}\right)\right)\right), w\left(\eta\left(t, x_{0}\right)\right) d t \geqslant \sigma \geqslant \varepsilon
\end{aligned}
$$

which contradicts (4). This proves assertion (iii) and the proof is complete.

\section{SOME CRITICAL POINT THEOREMS}

In this section, we give some applications of Lemma 1.1.

Let $Q \subset X$ be a Banach manifold with boundary $\partial Q$ and let $S$ be a closed set in $X$. We say that $\partial Q$ is linking with $S$ if $\partial Q \cap S=\emptyset$ and $\phi(Q) \cap S \neq \emptyset$ for any $\phi \in C(Q, X)$ with $\left.\phi\right|_{\theta Q}=$ id $\left.\right|_{\theta Q}$.

THEOREM 2.1. Suppose that $\partial Q$ is linking with $S, \sup _{x \in Q} f(x)<+\infty$, and that there is an $\alpha \in R^{1}$ such that

$$
f(x) \leqslant \alpha \text { for } x \in \partial Q ; f(x) \geqslant \alpha \text { for } x \in S .
$$

Define

$$
c=\inf _{\phi \in \Gamma} \sup _{x \in Q} f(\phi(x)) \text {, }
$$

where $\Gamma=\left\{\phi \in C(Q, X):\left.\phi\right|_{\partial Q}=\left.i d\right|_{\partial Q}\right\}$. Then we have

$$
\begin{aligned}
\text { (i) } & c \geqslant \alpha \text {; } \\
\text { (ii) } & K_{c} \backslash \partial Q \neq \emptyset \text {; } \\
\text { (iii) } & K_{c} \cap S \neq \emptyset \text { if } c=\alpha .
\end{aligned}
$$

Proof: $c \geqslant \alpha$ is evident. If $c>\alpha$, from the well-known deformation lemma ([4]; see also [1], [3]), we can easily prove that $K_{c} \backslash \partial Q=K_{c} \neq \emptyset$ (see [3]). If $c=\alpha$, clearly it is enough to prove $K_{c} \cap S \neq \emptyset$. Suppose by contradiction that $K_{c} \cap S=\emptyset$. Then using Lemma 1.1 to $-f(x)$ with $c=-c, A=\partial Q$ and $B=S$, we find an $\varepsilon>0$ and a homeomorphism $\eta$ of $X$ onto $X$ such that

$$
\eta x=x \text { for } x \in \partial Q:-f(\eta x) \leqslant-c-\varepsilon \text { for } x \in S .
$$

From the definition of $c$ we can find a $\phi_{0} \in \Gamma$ such that

$$
f\left(\phi_{0}(x)\right)<c+\varepsilon \text { for } x \in Q \text {. }
$$

Now let $\phi_{1}(x)=\eta^{-1}\left(\phi_{0}(x)\right)$; evidently $\phi_{1}: Q \rightarrow X$ is continuous. Since $\phi_{0}(x)=x$ for $x \in \partial Q$, we have $\phi_{1}(x)=\eta^{-1}\left(\phi_{0}(x)\right)=\eta^{-1} x=x$ for $x \in \partial Q$. Since $\partial Q$ is linking with $S$, we have $\phi_{1}(Q) \cap S \neq \emptyset$. Hence there exists $x_{0} \in Q$ such that $\phi_{1}\left(x_{0}\right) \in S$. This and (6) yield $f\left(\phi_{0}\left(x_{0}\right)\right)=f\left(\eta \phi_{1}\left(x_{0}\right)\right) \geqslant c+\varepsilon$, a contradiction to (7). The proof is complete. 
REMARK 2.1. (a) When condtion (5) is replaced by

$$
f(x) \leqslant \alpha \text { for } x \in \partial Q ; f(x) \geqslant \beta \text { for } x \in S ; \alpha<\beta,
$$

Theorem 2.1 becomes a well-known result (see, for example, [3] and [7]) which contains in particular the Mountain Pass Lemma ([1]) as well as some other useful critical point theorems (see [10]) as special cases.

(b) In the Mountain Pass setting, Theorem 2.1 was proved independently in [10] and [6]; see also [5], [8] and [9] for related results.

(c) If $Q$ is a compact set, Theorem 2.1 can be proved entirely by Lemma 1.1. More precisely, the case $c>\alpha$ can also be proved by using Lemma 1.1 instead of the old one. In fact, let $S_{1}=f^{-1}(c)$; we can easily prove that $\partial Q$ is linking with $S_{1}$ in this situation. Then since $\partial Q \subset f_{c}$ and $S_{1} \subset f^{c}$, we can show as above that $K_{c} \cap S_{1} \neq \emptyset$.

ThEOREM 2.2. Let the conditions of Theorem 2.1 be satisfied. Define

$$
d=\sup _{h \in \Gamma^{*}} \inf _{x \in S} f(h(x))
$$

where $\Gamma^{*}=\left\{h \in C(X, X): h\right.$ is a homeomorphism of $X$ onto $X$ with $\left.\left.h\right|_{\theta Q}=\left.i d\right|_{\theta Q}\right\}$. Then we have

$$
\begin{aligned}
& \text { (i) } \alpha \leqslant d \leqslant c ; \\
& \text { (ii) } K_{d} \backslash \partial Q \neq \emptyset \text {; } \\
& \text { (iii) } K_{d} \cap S \neq \emptyset \text { if } d=\alpha ;
\end{aligned}
$$

where $c$ is defined as in Theorem 2.1.

Proof: It is easy to see that $\alpha \leqslant d \leqslant c$. If $d>\alpha$, it can be proved by the wellknown deformation lemma that $K_{d}=K_{d} \backslash \partial Q \neq \emptyset$ (see, for example, [3]). If $d=\alpha$ and $K_{d} \cap S=\emptyset$, then use Lemma 1.1 to $-f(x)$ with $c=-d, A=\partial Q$ and $B=S$; we can find an $\varepsilon>0$ and a homeomorphism $\eta$ of $X$ onto $X$ such that

$$
\eta x=x \text { for } x \in \partial Q \text { and }-f(\eta x) \leqslant-d-\varepsilon \text { for } x \in S .
$$

Let $h_{0}(x)=\eta x$; evidently $h_{0} \in \Gamma^{*}$ and $\inf _{x \in S} f\left(h_{0}(x)\right)=\inf _{x \in S} f(\eta x) \geqslant d+\varepsilon$, and this contradicts the definition of $d$. Hence $K_{d} \cap S \neq \emptyset$ if $d=\alpha$. This completes the proof.

REMARK 2.2. (a) Under condition (8), Theorem 2.2 was proved in [3].

(b) Theorem 2.1 can be easily deduced from Theorem 2.2. In fact, if $c=\alpha$, we must have $d=c=\alpha$ and hence $K_{c} \cap S=K_{d} \cap S \neq \emptyset$ by Theorem 2.2. 
Corollary 2.1. Under the assumptions of Theorem 2.1, if condition (5) is replaced by

$$
f(x) \leqslant \alpha \text { for } x \in \partial Q \text { and } f(x)>\alpha \text { for } x \in S
$$

then we have

(i) $\alpha<d \leqslant c$,

(ii) $K_{c} \neq \emptyset, K_{d} \neq \emptyset$,

where $c$ and $d$ are defined as before.

REMARK 2.3. Corollary 2.1 improves a result in [3] where the conclusion was proved under the condition that $f \in C^{2-0}$ and that every critical value of $f$ corresponds to finitely many critical points.

REMARK 2.4. The idea of this paper can be used to improve several critical point theorems of even functionals as well. This and some variants of Theorem 2.1 can be used to deal with some new situations in elliptic boundary value problems. These will be done in a forthcoming paper.

\section{References}

[1] A. Ambrosetti and P.H. Rabinowitz, 'Dual variational methods in critical point theory and applications', J. Funct. Anal. 14 (1973), 349-381.

[2] V. Benci and P.H. Rabinowitz, 'Critical point theorems for indefinite functionals', Invent. Math. 52 (1979), 241-273.

[3] K.C. Chang, Critical Point Theory and Its Applications, (in Chinese) (Shanghai Scientific and Technical Press, 1986).

[4] D.C. Clark, 'A variant of Lusternik-Schnirelman theory', Indiana Univ. Math. J. 22 (1972), 65-74.

[5] D.G. de Figueiredo and S. Solimini, 'A variational approach to superlinear elliptic problems', Comm. Partial Differential Equations 9 (1984), 699-717.

[6] Dajun Guo, Jinxian Sun and Guijie Qi, 'Some extensions of the Mountain Pass Lemma', Differential and Integral Equations 1 (1988), 351-358.

[7] W.M. Ni, 'Some minimax principles and their applications in nonlinear elliptic equations', J. d'Analyse Math. 37 (1980), 248-275.

[8] P. Pucci and J. Serrin, 'Extensions of the mountain pass lemma', J. Funct. Anal. 59 (1984), 185-210.

[9] P. Pucci and J. Serrin, 'A mountain pass lemma', J. Differential Equations 60 (1985), 142-149.

[10] P.H. Rabinositz, 'Some aspects of critical point theory', in Proc. DD3, pp. 185-232 (Science Press, Beijing, 1986). 
Department of Mathematics Shandong University

Jinan

China
Present address:

Department of Mathematics

Heriot-Watt University

Riccarton, Edinburgh EH14 4AS

United Kingdom 